\title{
Assessing the Knowledge of Environmental Risk Factors for Cancer among the UAE Population: A Pilot Study
}

\author{
Samrein B.M. Ahmed ${ }^{1,2, *}$, Sara Amer ${ }^{1}$, Amal Hussein ${ }^{1} \oplus$, Drishti D. Kampani ${ }^{1}$, \\ Nour Al Hasham ${ }^{1}$, Mohamed M. Assker ${ }^{1}$, Nour Shawa ${ }^{2}$, Dima Saleh ${ }^{1}$ and Khalid O. Alfarouk ${ }^{3}$ \\ 1 College of medicine, University of Sharjah, Sharjah 27272, UAE; MD_Saleema@outlook.com (S.A.); \\ amalmh@sharjah.ac.ae (A.H.); U16103939@sharjah.ac.ae (D.D.K.); Nour_alhasham@outlook.com (N.A.H.); \\ U17103019@sharjah.ac.ae (M.M.A.); U17100648@sharjah.ac.ae (D.S.) \\ 2 Sharjah Institute for Medical Research, University of Sharjah, Sharjah 27272, UAE; \\ nourshawa.1994@gmail.com \\ 3 Alfarouk Biomedical Research LLC, Temple Terrace, FL 33617, USA; khaliomer@gmail.com \\ * Correspondence: samahmed@sharjah.ac.ae
}

Received: 14 April 2020; Accepted: 22 April 2020; Published: 25 April 2020

check for updates

\begin{abstract}
The incidence of cancer is increasing worldwide as well as in the United Arab Emirates (UAE). Currently, researchers are advocating not only for prevention programs but also for early detection. In this study, we aimed to assess the general awareness of cancer among the UAE population, with a focus on environmental risk factors. A descriptive cross-sectional design was employed, and a structured questionnaire was used to collect data from 385 participants. A total of $91.2 \%$ of the study population identified cancer as the leading cause of death, while $64.6 \%$ of the subjects were able to identify the key causes of cancer. A total of $87.3 \%$ and $70.5 \%$ of the participants were able to define tobacco and alcohol, respectively, as cancer-causing agents. Most of the study population failed to identify cancer-related infectious agents and incense smoke as carcinogens. Respondents in the medical professions had the highest knowledge score when compared with respondents with a non-medical profession and unemployed participants $(p<0.0005)$. To fill the gaps in cancer-related knowledge, participants were asked about their preferred method for cancer education, and $83.9 \%$ of the participants favored the media as a source of information. Conclusively, our findings indicated a gap in cancer knowledge among UAE residents, which highlights the importance of educational campaigns by health authorities; a follow-up study evaluating the success of educational campaigns is also warranted.
\end{abstract}

Keywords: cancer; environment; risk factors; UAE population; knowledge

\section{Background}

Cancer is one of the leading causes of mortality worldwide. Global estimates show that the total incidence of cancer was 18.1 million with 9.6 million deaths in 2018, making it an important public health concern [1]. In the United Arab Emirates (UAE) alone, cancer is the third leading cause of death, preceded by cardiovascular disease and injury [2]. Despite advancements and improved clinical approaches to cancer treatment, resistance of cancer cells is inevitable. Therefore, scientists are proposing to combat cancer by either refining screening methods or developing protection strategies against cancer-causing agents [3].

Cancer etiology is attributed to (a) non-preventable genetic factors and (b) preventable environmental factors. These factors include occupational exposure, infectious diseases, air pollution, 
and ultraviolet (UV) radiation, among others. The substances that contribute to cancer development are called carcinogens, which act by damaging DNA and altering cellular machinery [4].

The significant increase in cancer burden worldwide has been linked to several factors, including an increase in cancer-causing behaviors and frequent exposure to cancer-causing agents. The World Health Organization (WHO) states that $30 \%-50 \%$ of cancers can be prevented by avoiding exposure to a carcinogen, adopting a healthier lifestyle and implementing evidence-based prevention strategies [5]. In the UAE, according to information published on the UAE governmental portal, approximately 4500 new cancer cases are reported each year [6].

Due to changes in economic and lifestyle trends, many disease patterns have shifted, including those of cancer. Primary prevention through environmental interventions has become the mainstay for reducing cancer burden. Cessation of tobacco and alcohol consumption remains the most common prevention recommendation worldwide [7]. Additionally, a case-control study by Tse and coinvestigators linked incense smoke to lung cancer [8]. Other important carcinogens that substantially influence cancer risk include bisphenol A, phthalates and naphthalene [9-11]. Moreover, commonly found substances such as household cleaning products, plastic containers, indoor combustion, soot and wood dust are reportedly carcinogenic [12].

Diet is also thought to play a crucial role in cancer development. Various food contaminants and food components have been linked to cancer pathogenesis. Examples of these carcinogens include aflatoxin [13,14] and processed meat [15]. Acrylamide is a dietary compound that is considered to have carcinogenic potential, and its mutational signature has been reported [16]. In addition, evidence from rats and mice indicated that saccharin has carcinogenic effects [17]. However, the carcinogenic potential of food-related agents depends on the individual's susceptibility to developing cancer and their level of exposure.

It is estimated that occupational hazards can expose individuals to more than half of the chemicals classified as carcinogens. Based on the data provided by the Cancer Research UK, $4 \%$ of cancers are due to health hazards at work. Additionally, according to the International Agency for Research on Cancer, over 120 chemical or biological agents are classified as carcinogens [18]. Among these carcinogens are diesel exhaust, nickel, pesticides, asbestos, silica, wood dust and solar radiation [19]. Some common cancers associated with occupational carcinogen exposure are leukemia and lung cancer $[20,21]$.

In addition to the abovementioned environmental carcinogens, biological carcinogens such as human papilloma virus, Epstein-Barr virus, hepatitis B and C and Helicobacter pylori have been associated with cancer incidence [22-24].

In the UAE, several programs, including the National Periodic Health Screening Program and Cancer Screening Initiative, were launched in 2015 by the Ministry of Health and Prevention to increase public awareness of cancer and the importance of its early detection through regular medical check-ups for more effective treatments [25]. However, little is known about the knowledge level or attitudes of the public towards cancer risk factors and exposure to carcinogens.

Because the UAE has stated measures regarding health safety for different occupations [26,27], in the present study, we aimed to assess the knowledge of the United Arab Emirates general population regarding environmental carcinogens. This will ultimately help in increasing the UAE community's awareness about environmental carcinogens and thus help to establish goal-oriented campaigns that aim to reduce cancer burden locally by targeting and minimizing weaknesses in public knowledge. Additionally, we aimed to assess the knowledge and awareness of cancer among UAE populations with various educational levels, different age groups, and diverse occupational backgrounds.

\section{Materials and Methods}

To estimate the prevalence of knowledge regarding environmental risk factors among our study population, a descriptive cross-sectional design was used. The inclusion criteria included UAE residents aged 18 and above who were able to communicate in either English or Arabic, which are the most common languages in the UAE. Adults over the age of 18 who neither resided in the UAE at the 
time of the study, nor spoke English or Arabic were excluded from the study. The recruitment of the participants took place from February-May 2019.

A nonprobability convenience method was used to recruit subjects from public areas, such as parks, malls, and residential compounds. Because no similar study was conducted in the region, an expected proportion of study participants knowledgeable about the environmental risk factors for cancer was set at $50 \%$ to achieve the maximum sample size. A margin error of $5 \%$ was considered for the sample size calculation. The following equation was employed to calculate the sample size: $n=\frac{(4 p(1-p))}{S E^{2}}$, where $n$ is the sample size, $p$ is the expected prevalence and SE is the sampling error. Accordingly, 385 subjects were targeted.

A self-administered structured questionnaire was developed based on information acquired from identified sources and published cancer factors as well as global and local statistics, which are all cited in this manuscript. The questionnaire included a total of 41 questions. The questionnaire included items related to demographics (6), general knowledge of cancer (5), knowledge related to environmental carcinogens (27), sources of cancer-related knowledge (2) and knowledge related to cancer prevention (1). The questionnaire was self-administered, and the data collectors were available to clarify any ambiguity. The questionnaire was also pilot tested to ensure face and content validity.

Prior to data collection, the data collectors ensured that the method of data collection was consistent and standardized. The data collectors verbally explained the project and its outcomes to the participants. Moreover, it was clearly stated in the information sheet as well as verbally that completing the questionnaire indicates the agreement to join the study. No signature was taken to ensure the anonymity of the study. Additionally, the data collector explained that the questionnaire would take only $10 \mathrm{~min}$ to complete; the time was allotted after the pilot testing. The collected data were available only to the investigators to ensure confidentiality. This study was approved by the Research Ethics Committee at the University of Sharjah (Ref. No. REC-18-12-02-01) before the study commenced.

After data entry and cleaning, 32 questionnaires were excluded because of incomplete and missing data. The data were analyzed electronically using IBM SPSS, version 25 (IBM Corp., Armonk, NY, USA). The study subgroups included age, level of education, occupation field, country of origin, UAE residence duration, and residence in different emirates. Categorical data are summarized as frequencies and percentages, and scale data are summarized as the mean and standard deviation when normally distributed and the median and interquartile range when skewed. The normality of the scale data was tested visually using Q_Q plots and statistically using the Kolmogorov-Smirnov test. Parametric statistical tests such as Analysis of Variance (ANOVA) test and Student's $t$-test were performed to compare mean knowledge scores between groups when parametric assumptions were met. Otherwise, the Kruskal-Wallis and Mann-Whitney U test non-parametric tests were performed when knowledge score data showed a skewed distribution in at least one of the compared groups. Stepwise multiple linear regression (MLR) was performed to identify significant predictors of the cancer-related knowledge score. Variables entered in the model were defined as those associated with the dependent variable in the bivariate analysis, with a $p$-value less than or equal to 0.20 . Assumptions of MLR were all tested prior to performing the analysis. Collinearity was tested using tolerance and variance inflation factor, and multivariate outliers were checked using Mahalanobis distance. Equality of variance was tested using Levene's test. Dummy variables were created for categorical variables with more than two groups. A $p$-value less than 0.05 was considered statistically significant.

\section{Results}

\subsection{Demographic Data of the Study Population}

In this study, 353 participants were included in our final analysis. Age was categorized into 4 groups: $18-24$ years of age (39.4\%), 25-35 years of age (23.2\%), 36-45 years of age (17.6\%), and above 45 years of age $(19.8 \%)$. Of all study participants, $84.4 \%$ had bachelor's degrees or above, while $15.6 \%$ completed elementary, intermediate, or high school education. Of the participants, $72.5 \%$ were 
employed, $12.5 \%$ were employees in the medical field, and $64 \%$ were employed in a non-medical field. Most of the study population had been UAE residents for more than 5 years $(82.4 \%, n=291)$. Participants resided in the seven emirates of the UAE, where the highest portion (43.1\%) of the study sample resided in Sharjah.

\subsection{Assessing the Study Population's General Knowledge about Cancer}

Of the participants, $98.3 \%(n=347)$ had heard of cancer, of whom $92.8 \%(n=322)$ believed that cancer could lead to death, and $81.6 \%(n=283)$ considered cancer to be a preventable disease.

Figures 1 and 2 illustrate the general knowledge and awareness of cancer among the study population. Two thirds of the study subjects were correctly aware that cancers can be caused by both genetic and environmental factors (Figure 1).

General causes of cancer identified by the participants

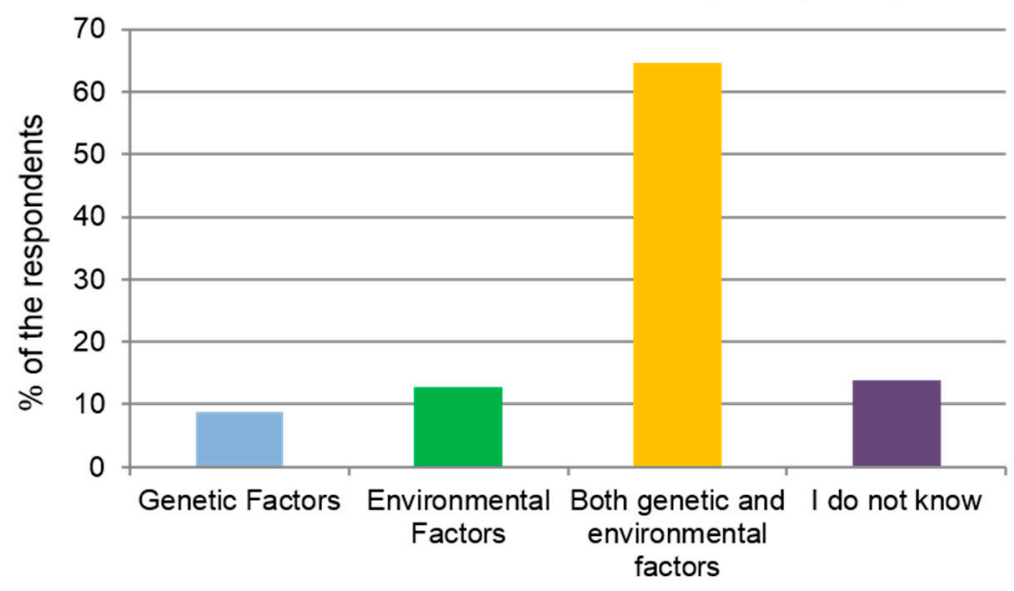

Figure 1. General causes of cancer identified by the study population.

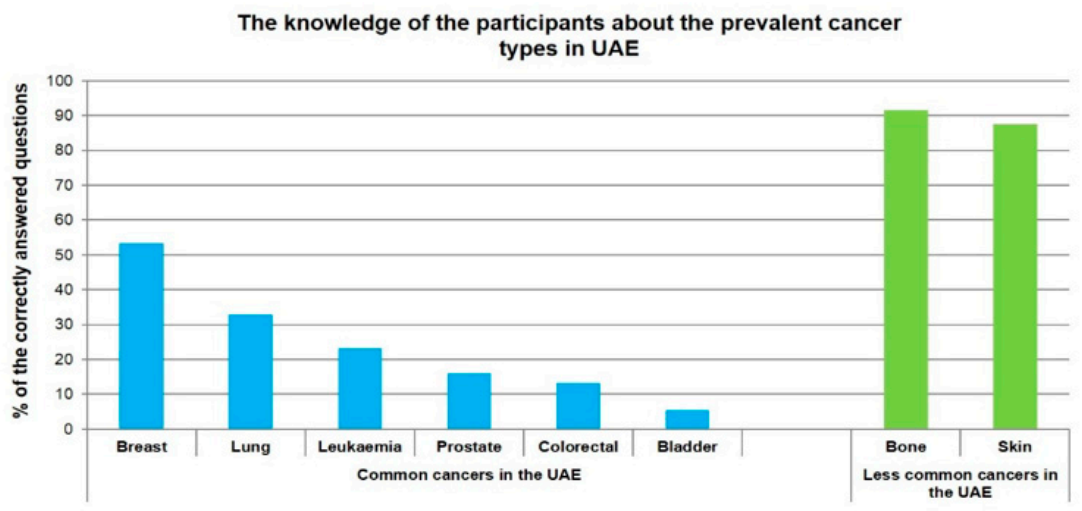

Figure 2. The knowledge of the participants regarding the prevalent cancer types in the UAE.

According to the WHO 2018 statistics, breast, colorectal, prostate, leukemia, bladder and lung cancers are among the most prevalent cancers in the UAE [28]. Upon questioning the participants about the predominant types of cancer in the UAE, $26.6 \%(n=94)$ of the respondents stated that they did not know about cancer prevalence in the country. Out of the six common types of cancer in UAE, breast cancer was correctly identified as common by more than half the respondents. Leukemia, prostate, and colorectal cancer were least recognized as prevalent cancers with only $23.2 \%, 15.9 \%$ and $13.3 \%$ identifying them, respectively. Despite the poor knowledge and awareness of prevalent cancers, the study population correctly identified the non-prevalent cancers, such as bone and skin cancers, as uncommon. Overall, the study population demonstrated a lack of knowledge regarding the prevalent cancers in the UAE. 


\subsection{Knowledge of Cancer Risk Factors among the Study Population}

Figure 3 demonstrates the level of knowledge about the leading risk factors for cancer. The impact of chemicals, lifestyle, and radiation factors on cancer development was correctly identified but poor knowledge was observed regarding tumor-associated microorganisms. Less than half of the subjects $(40.8 \%, n=144)$ believed that infections are a possible leading cause of cancer.

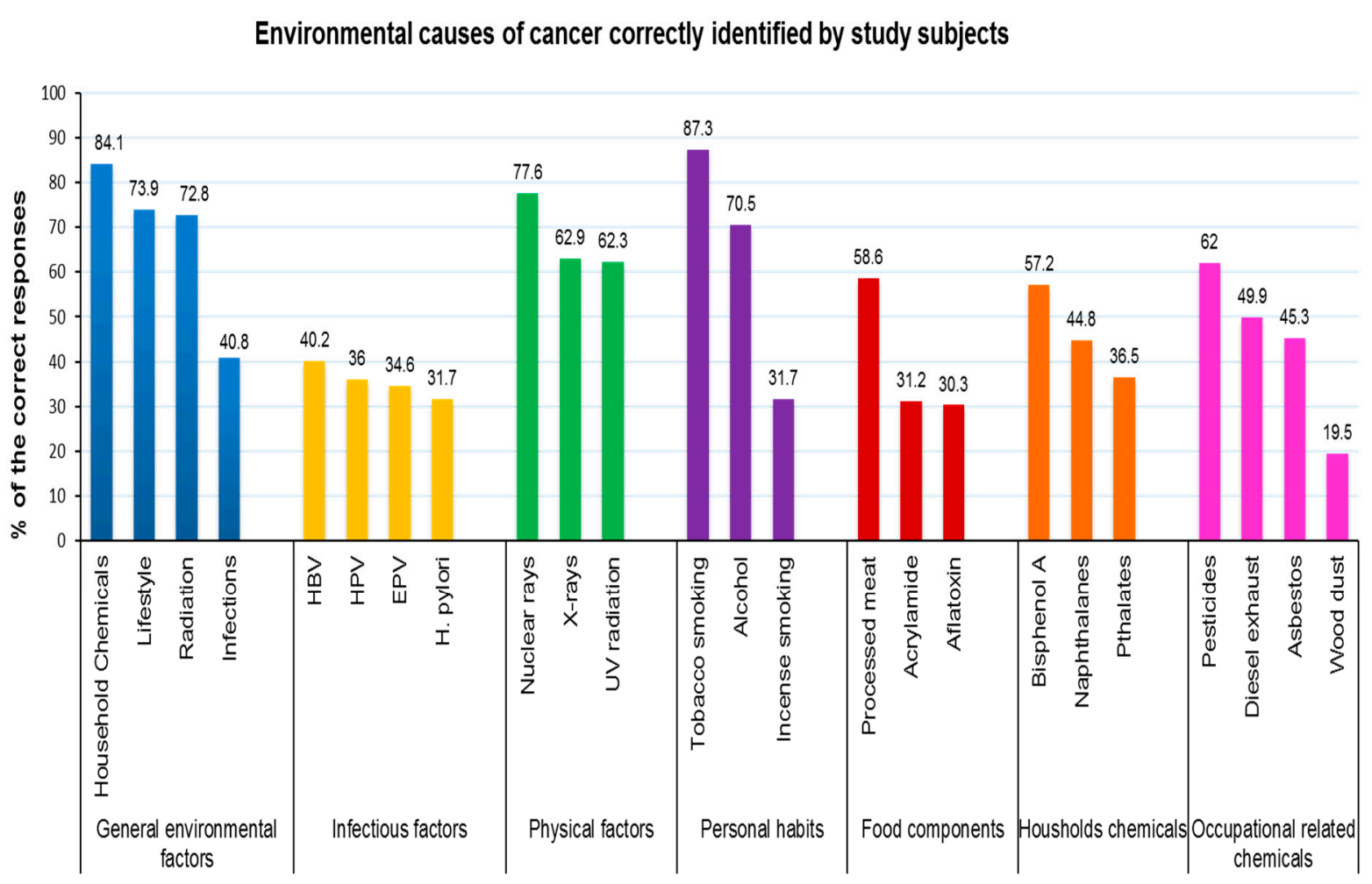

Figure 3. Environmental causes of cancer correctly identified by the study subjects. HBV: Hepatitis B virus; HPV: Human Papilloma virus; EPV: Epstein-Barr virus; H. pylori: Helicobacter pylori.

Additionally, low awareness was found among the respondents regarding the effects of some food-related components and contaminants, such as saccharin $(36.3 \%)$, aflatoxin $(30.3 \%)$ and acrylamide (31.2\%).

The best identified cancer causes by our population were physical factors and lifestyle causes. A total of $77.6 \%$ of the participants identified nuclear radiation as a risk for cancer development; tobacco smoking and alcohol consumption were positively identified as causes by $87.3 \%$ and $70.5 \%$ of the study population, respectively. Conclusively, many of the study participants were able to identify smoking, alcohol, and nuclear radiation as cancer-causing factors, but disappointingly, a large section of the respondents failed to categorize infections, some food-related contaminants/components, incense smoke, and occupation-associated carcinogens as risk factors.

\subsection{The Knowledge Scores among the Subgroups According to Different Study Variables}

The cancer-related knowledge score of all study participants ranged from 0 to $100 \%$, with a mean of $51 \%(S D=20.5)$ and a median of $52 \%(I Q R=25.9)$. The score had a non-normal distribution as indicated by the Kolmogorov-Smirnov test $(\mathrm{KS}=0.054, \mathrm{df}=353, p$-value $=0.016)$. Knowledge scores were compared between the different groups of the study variables (age, level of education, occupation, or residency). The two variables that were significantly associated with the cancer-related knowledge score were occupation and duration of residency in the UAE. Respondents in the medical professions had the highest knowledge score $(64.2 \%)$ compared with those of participants with non-medical professions $(50.6 \%)$ and unemployed respondents $(45.3 \%)(p<0.0005)$. 
Those who were UAE residents for less than 5 years showed lower cancer-related knowledge than those who resided in the UAE for more than 5 years $(44.4 \%$ and $51.9 \%$, respectively, $p=0.012)$. None of the other variables, including age, education, country of origin, and Emirate, showed a significant relationship with the cancer-related knowledge score (Table 1).

Table 1. Cancer-related Knowledge Score by Demographic characteristics of study participants.

\begin{tabular}{|c|c|c|c|c|}
\hline & $n$ & Score $(\%)$ & Test Value & $p$-Value \\
\hline \multicolumn{5}{|l|}{ Age } \\
\hline $18-24$ & 139 & 51.9 & \multirow{4}{*}{$6.681 *$} & \multirow{4}{*}{0.083} \\
\hline $25-35$ & 82 & 48.1 & & \\
\hline $36-45$ & 62 & 48.1 & & \\
\hline$>45$ & 70 & 55.6 & & \\
\hline \multicolumn{5}{|l|}{ Level of education } \\
\hline Below University & 55 & 48.1 & \multirow{2}{*}{$7015.0 *$} & \multirow{2}{*}{0.089} \\
\hline University and Above & 298 & 51.9 & & \\
\hline \multicolumn{5}{|l|}{ Occupational field } \\
\hline Medical & 44 & 64.2 & \multirow{3}{*}{$13.264^{* *}$} & \multirow{3}{*}{$<0.0005$} \\
\hline Non-Medical & 226 & 50.6 & & \\
\hline Unemployed & 83 & 45.3 & & \\
\hline \multicolumn{5}{|l|}{ Country of origin } \\
\hline Middle East & 275 & 51.9 & \multirow{2}{*}{10635 * } & \multirow{2}{*}{0.910} \\
\hline Others & 78 & 48.1 & & \\
\hline \multicolumn{5}{|l|}{ UAE Residency duration } \\
\hline Less than 5 years & 62 & 44.4 & \multirow{2}{*}{$7187.0 *$} & \multirow{2}{*}{0.012} \\
\hline 5 years and above & 291 & 51.9 & & \\
\hline \multicolumn{5}{|l|}{ Emirate } \\
\hline Abu Dhabi & 53 & 48.1 & \multirow{4}{*}{1.127 * } & \multirow{4}{*}{0.770} \\
\hline Dubai & 78 & 51.9 & & \\
\hline Sharjah & 152 & 48.1 & & \\
\hline Northern Emirates & 70 & 51.9 & & \\
\hline
\end{tabular}

The stepwise MLR model included the variables age, level of education, occupation, and residency duration in the UAE. The model was significant in predicting $7.8 \%$ of the total variability in the cancer-related knowledge score $(\mathrm{F}=14.717, p<0.0005)$. Two predictors were identified by the regression model: occupation and age. Having a medical occupation predicted an increase in the knowledge score of $15.2 \%$ compared to the scores of those who were unemployed $(p<0.0005)$, while being above 45 years old was associated with a $7.0 \%$ increase in the knowledge score compared to the scores of those who were 18 to 24 years old ( $p=0.008$ ), when keeping all other variables constant. Accordingly, it was inferred that participants with a medical profession positively impacted the knowledge score of the study population, while surprisingly, participants with more than 5 years of residence in the UAE exhibited better knowledge than those who had been in the UAE for fewer than 5 years.

\subsection{Sources of Cancer Knowledge}

Most of the participants $(81.9 \%)$ considered the sources of media, such as TV, radio, and internet, to be their source of knowledge, while a small percentage of the subjects $(20.7 \%)$ claimed that they obtained health-related information from health care centers. To determine the best method to increase cancer knowledge among the UAE population, the participants were asked what source they would prefer to use to increase their knowledge of cancer. Among the media, campaigns and hospitals, 
$83.9 \%$ of participants selected the media as their preferred source of knowledge, followed by campaigns and hospitals.

\subsection{Desirable Methods to Prevent Poor Cancer Outcomes Identified by the Study Participants}

We were also interested in assessing the awareness of the study population regarding different cancer prevention approaches. Regular medical check-ups and healthy lifestyle and diet were the most popular methods among our respondents (67.4\% and $59.8 \%$, respectively). However, just under $27 \%$ of the respondents thought that vaccines could help with cancer prevention.

\section{Discussion}

Various environmental factors that are related to lifestyle, personal habits, food and occupation have been found to be linked with cancer development [5]. Exposure to certain environmental factors related to cancer development is considered a modifiable cancer risk factor [5]. Moreover, since cancer treatment is challenging for advanced-stage cancer, early diagnosis as well as the enhancement of preventive measures against cancer are a prerequisite to improve disease outcomes [3]. In this pilot study, we aimed to assess the general knowledge about cancer as well as to assess the awareness of the UAE population regarding some of the identified environmental risk factors using a cross-sectional study design. More than half of the study participants were able to relate cancer development to both environmental and genetic factors. Although the study population was aware of some of the environmental risk factors, they exhibited a lack of knowledge about others, which indicates that further comprehensive studies are required to develop and promote existing awareness programs.

\subsection{Knowledge of Cancer as a Deadly Disease Is High}

In this study, cancer was identified as a leading cause of death by $91.2 \%$ of the participants. This finding is consistent with previously published studies reporting that general populations strongly believe that cancer is a fatal disease, particularly in the Middle Eastern region, where a diagnosis of cancer is highly stigmatized $[29,30]$.

Furthermore, a study conducted in the US further confirmed this observation, in which people held strongly fatalistic beliefs about cancer. These beliefs have caused people to disengage from cancer prevention strategies because they believe that nothing can be done to prevent it [30]. These attitudes may have led to an increase in cancer incidence. Additionally, people are less likely to participate in screening tests for fear of a positive result [31].

Another study conducted in Ireland concluded that $15 \%$ of the study population agreed with the statement that if cancer was present in their family, "there was nothing a person could do to reduce their own personal cancer risk" [32].

On a positive note, although unexpected, $80.2 \%$ of the respondents concurred that cancer was a preventable disease, indicating that the public was familiar with awareness campaigns.

\subsection{Knowledge of Common Cancers among the Study Population}

In this study, we found that $53.5 \%$ of the participants correctly responded that breast cancer is a common type of cancer. This result is comparable to findings from a previous study in which UAE female residents reported having limited knowledge of breast cancer screening tests [33]. Despite the Pink Caravan campaign initiative [34], public awareness requires further improvement.

Regrettably, our study population showed a lack of knowledge when asked about other types of cancer. For example, only $13.3 \%(n=47)$ of the respondents agreed that colorectal cancer is a common cancer in the UAE, despite its predominance in males in the UAE [28]. In September 2018, the Ministry of Health-UAE launched a colorectal cancer (CRC) awareness program for early detection and prevention [35]. However, our study sample recruitment was performed in the first half of 2019, which might explain the lack of awareness about CRC. This might indicate the importance of a future follow-up study to evaluate the success of the awareness campaign. 
An important question about cancer awareness is how to identify the people with the least amount of knowledge. In this study, the respondents whose professions were related to the medical field showed better knowledge scores than the rest of the respondents $(p<0.0005)$, implying a need to diversify health awareness campaigns to appeal to people from different backgrounds. This finding is in agreement with an Indian study that reported that women in the medical field have better breast cancer awareness than women employed in non-medical professions when both groups had received the same level of education [36].

Additionally, it is worth noting that the level of education had no correlation with the cancer knowledge score. This result implies that participants with lower educational backgrounds have comparable knowledge to those with higher education backgrounds. A study by Gurdal et al. inferred that women are required to increase their awareness about breast cancer regardless of their level of education [37]. One recommendation would be to consider the introduction of cancer-oriented awareness campaigns at all levels for better coverage. Accordingly, health care facilities as well as the government need to work together to promote awareness of other common types of cancer that are prevalent in the country.

\subsection{Knowledge of Environmental Risk Factors}

In this study, more than half of the study population (64.6\%) agreed that cancer is caused by both genetic and environmental factors. Interestingly, previous studies have indicated that people strongly emphasize only family history and genetic factors as causes of cancer [38,39]. In addition, our findings demonstrate that participants were able to correctly report some environmental factors.

A study conducted in England reported that $91 \%$ of the study population was able to correctly report cancer risk factors [38]. Similarly, in an American study, the population sample identified cancer risk factors, yet a lack of knowledge regarding the association between the major risk factor and the corresponding cancer was noted in the study [39]. These two studies showed better cancer-related knowledge in some of the Western countries than that of our study population. This indeed indicates that more effort is required to promote cancer-related knowledge among UAE residents, even though more than half of the study population was able to relate both genetic and environmental factors to cancer.

Infections were the least recognized cancer risk factor in the study population. This observation is consistent with a study conducted in Latin America, in which $47 \%$ of the participants were able to identify Human papillomavirus (HPV) as a risk factor for cancer [40]. In contrast, a study in Japan demonstrated that the public had better knowledge of cancer-associated infections as a risk factor for cancer than of other risk factors [41]. Thus, it is worth increasing awareness about infectious agents as a cause of cancer, particularly HPV, because it was recently reported that cervical cancer-related deaths are increasing in the UAE [42].

In our study population, smoking $(87.3 \%)$ and alcohol consumption $(70.5 \%)$ were primarily identified as environmental risk factors for cancer. In contrast, a previous report that was conducted in Germany showed that only $47 \%$ of the study population was able to link alcohol to cancer [43]. The better knowledge in our study population could be because of the sociocultural stigma associated with alcohol consumption in the region. This indeed discourages the practice and promotes the idea that alcohol is unhealthy [44]. The expected relatively high knowledge of the link between smoking and cancer is due to the issued federal law No. 15 of 2009 regarding tobacco control, which was agreed upon by the government and departments of health and smoking cessation clinics that are easily accessible across the country [45]. Moreover, the anti-smoking campaign agenda was announced in 2010 by hospitals and the UAE Ministry of Health targeting the whole population of the UAE [46,47]. It is worth noting that the health hazards of smoking are a global concern, and all sources of media demonstrate this explicitly, which might explain the noted level of smoking-related knowledge among the participants. Consistent with our study, a previous Polish study on tobacco health risks revealed that $92 \%$ of the study participants recognized the link between tobacco smoking and cancer [48]. 
Expectedly, incense smoke (bakhour) was least identified as a possible cancer-causing agent in our study population (31.7\%). This result highlights the fact that although people are aware of common risk factors, it is important to educate the general public about some traditional practices that might lead to cancer. An interesting study by Yvonne et al. showed that the burning of indoor incense was associated with oral microbiota composition among 303 Emirati adults [49], which implies the effect of incense smoke on the health of the Emirati population. Although this study highlighted the negative effect of incense smoke on the health of the Emirati population, no studies have demonstrated the link of leukemia and lung cancer, two of the most prevalent cancers in the UAE, with household incense. Performing such a study will help in providing evidence of the link between the use of the incense and cancer development among the UAE population. Hence, preventive health measures can be issued by the health authority to enhance awareness about incense smoke as a possible risk of cancer among the UAE population.

Furthermore, the study population demonstrated a lack of knowledge regarding carcinogenic food contaminants/additives such as saccharine, pesticides, bisphenol A, aflatoxin, and acrylamide. Assessing population awareness of exposure to pesticides among other chemical carcinogens in the Hail region-KSA revealed an extreme lack of awareness that made an awareness campaign a necessity in the Hail region [50].

\subsection{Sources of Knowledge and Methods to Prevent Poor Cancer Outcomes}

We found that our study population favored being educated about health and prevention via the media. A study on the use of social media to raise awareness about breast cancer suggested that social media is a preferable method for raising breast cancer awareness [51]. Having mentioned this, the employment of the media should be tailored to the age group, level of education, language, and cultural barriers. Moreover, because almost half of the study participants were between 18 and 35 years of age, it was not surprising that the media was the dominant choice for gaining knowledge.

Counterintuitively, our results showed that a residency of more than 5 years in the UAE was associated with a higher knowledge score $(p=0.012)$. This result may be explained in part by the fact that the major health authorities in the UAE started intensive cancer awareness campaigns in 2013 [52]. The effectiveness and evaluation of the launched awareness programs and campaigns require further detailed studies that is beyond the scope of this study.

Regular medical check-ups and living a healthy lifestyle were considered methods to reduce poor cancer outcomes by more than half of the study population. On the other hand, most of the study population $(74 \%)$ did not consider vaccination to be a preventive measure against cancer. Nevertheless, this finding is consistent with the demonstrated lack of knowledge regarding cancer-related infections. This further emphasizes the need to develop campaigns to raise awareness among the population regarding the microorganisms associated with cancer development, particularly cervical cancer, as its incidence is increasing in the UAE [42].

\subsection{Study Limitations}

This research used a convenience sampling method, which may affect the generalizability of the results. In the conservative society of the UAE, home visits are not appropriate, and physical mail is not a popular mode of communication. Thus, the sex demographics of the respondents were not ascertained in this study. In addition, the age demographic of the study may not be perfectly representative of the UAE population. According to 2018 UAE population estimates, $42.17 \%$ of the total adult population is between 25 and 34 years of age; however, in this study, only $23.2 \%$ of the participants were in this age range. Thus, our findings may underestimate the magnitude of age-related and education-related differences in the knowledge and attitudes related to common cancers and environmental risk factors related to cancer development. 


\section{Conclusions}

A lack of awareness about common yet risky exposure to substances may be an important and overlooked impediment to national cancer prevention strategies. The findings solidly support the development of intensive cancer-relevant information campaigns in educational institutions, workplaces, and society. These campaigns should particularly raise cancer awareness regarding cancer-associated infections as well as sources of indoor pollution, such as incense smoke, which is associated with increased cancer risk and cervical cancer, leukemia, and lung cancer, respectively. Further studies could assess the specific effects of community- and government-led cancer prevention initiatives on awareness levels. Future studies could also investigate whether sex differences exist in terms of the knowledge of common cancers and awareness of available screening programs. Moreover, the prevalence of carcinogenic exposure within society, in terms of duration and levels, could be investigated further.

Moreover, these campaigns and awareness programs should promote knowledge about the common cancers in the country and their available screening programs as well as their associated risk factors.

Author Contributions: S.B.M.A. contributed to the conception and design of the study and the questionnaire; S.B.M.A., S.A., A.H. and D.D.K. participated in writing and critically revising the manuscript; S.A. and A.H. organized the data and performed the statistical analysis; S.B.M.A. prepared the figures; S.A., N.A.H., M.M.A., D.S. and N.S. participated in data collection; A.H. contributed to the methodology section and provided valuable feedback. K.O.A. proofread the manuscript and provided valuable comments that enhanced the manuscript. All authors contributed to manuscript revision and read and approved the submitted version. All authors have read and agreed to the published version of the manuscript.

Funding: This study was funded by the University of Sharjah Research funding department (grant No 1801090143).

Conflicts of Interest: The authors declare that the research was conducted in the absence of any commercial or financial relationships that could be construed as a potential conflict of interest.

Participant Information Sheet (PIS): The PIS was presented upon approaching the participants. Agreement to fill the questionnaire indicated the agreement of the participants to join the study; this was clearly mentioned in the PIS.

Data Availability: All data generated or analyzed during this study are included in this published article.

\section{Abbreviations}

UV Ultraviolet radiation

DNA Deoxyribonucleic acid

UAE United Arab Emirates

UK United Kingdom

US United States

\section{References}

1. Ferlay, J.; Colombet, M.; Soerjomataram, I.; Mathers, C.; Parkin, D.M.; Piñeros, M.; Znaor, A.; Bray, F. Estimating the global cancer incidence and mortality in 2018: GLOBOCAN sources and methods. Int. J. Cancer 2018, 144, 1941-1953. [CrossRef] [PubMed]

2. Cancer Incidence in United Arab Emirates Annual Report of The Uae-National Cancer Registry-2014; Ministry of Health and Prevention: Abu Dhabi, United Arab Emirates, 2014.

3. Wardle, J.; A Robb, K.; Vernon, S.; Waller, J. Screening for prevention and early diagnosis of cancer. Am. Psychol. 2015, 70, 119-133. [CrossRef] [PubMed]

4. Migliore, L.; Coppedè, F. Genetic and environmental factors in cancer and neurodegenerative diseases. Mutat. Res. Mutat. Res. 2002, 512, 135-153. [CrossRef]

5. Cancer Prevention. Available online: https://www.who.int/cancer/prevention/en/ (accessed on 1 October 2019).

6. Cancer. Available online: https://government.ae/en/information-and-services/health-and-fitness/chronicdiseases-and-natural-disorders/cancer- (accessed on 1 October 2019). 
7. Shield, K.D.; Parkin, D.M.; Whiteman, D.C.; Rehm, J.; Viallon, V.; Micallef, C.M.; Vineis, P.; Rushton, L.; Bray, F.; Soerjomataram, I. Population Attributable and Preventable Fractions: Cancer Risk Factor Surveillance, and Cancer Policy Projection. Curr. Epidemiol. Rep. 2016, 3, 201-211. [CrossRef]

8. Tse, L.A.; Yu, I.T.; Qiu, H.; Au, J.S.K.; Wang, X.-R. A Case-Referent Study of Lung Cancer and Incense Smoke, Smoking, and Residential Radon in Chinese Men. Environ. Health Perspect. 2011, 119, 1641-1646. [CrossRef]

9. Konieczna, A.; Rutkowska, A.; Rachoń, D. Health risk of exposure to Bisphenol A (BPA). Rocz. Państwowego Zakładu Hig. 2015, 66, 5-11.

10. Wang, Y.-C.; Chen, H.-S.; Long, C.-Y.; Tsai, C.-F.; Hsieh, T.-H.; Hsu, C.; Tsai, E.-M. Possible mechanism of phthalates-induced tumorigenesis. Kaohsiung J. Med Sci. 2012, 28 (Suppl. 7), S22-S27. [CrossRef]

11. Kokel, D.; Li, Y.; Qin, J.; Xue, D. The nongenotoxic carcinogens naphthalene and para-dichlorobenzene suppress apoptosis in Caenorhabditis elegans. Nat. Methods 2006, 2, 338-345. [CrossRef]

12. Espina, C.; Straif, K.; Friis, S.; Kogevinas, M.; Saracci, R.; Vainio, H.; Schüz, J. European Code against Cancer 4th Edition: Environment, occupation and cancer. Cancer Epidemiol. 2015, 39 (Suppl. 1), S84-S92. [CrossRef]

13. Norat, T.; Scoccianti, C.; Boutron-Ruault, M.-C.; Anderson, A.; Berrino, F.; Cecchini, M.; Espina, C.; Key, T.; Leitzmann, M.; Powers, H.; et al. European Code against Cancer 4th Edition: Diet and cancer. Cancer Epidemiol. 2015, 39 (Suppl. 1), S56-S66. [CrossRef]

14. Marchese, S.; Polo, A.; Ariano, A.; Velotto, S.; Costantini, S.; Severino, L. Aflatoxin B1 and M1: Biological Properties and Their Involvement in Cancer Development. Toxins 2018, 10, 214. [CrossRef] [PubMed]

15. Lippi, G.; Mattiuzzi, C.; Cervellin, G. Meat consumption and cancer risk: A critical review of published meta-analyses. Crit. Rev. Oncol. 2016, 97, 1-14. [CrossRef] [PubMed]

16. Zhivagui, M.; Ng, A.W.; Ardin, M.; Churchwell, M.I.; Pandey, M.; Renard, C.; Villar, S.; Cahais, V.; Robitaille, A.; Bouaoun, L.; et al. Experimental and pan-cancer genome analyses reveal widespread contribution of acrylamide exposure to carcinogenesis in humans. Genome Res. 2019, 29, 521-531. [CrossRef]

17. Reuber, M.D. Carcinogenicity of saccharin. Environ. Health Perspect. 1978, 25, 173-200. [CrossRef] [PubMed]

18. Iarc Monographs on the Identification of Carcinogenic Hazards to Humans. Available online: https: //monographs.iarc.fr/agents-classified-by-the-iarc/ (accessed on 1 October 2019).

19. Cherrie, J.; Hutchings, S.; Ng, M.G.; Mistry, R.; Corden, C.; Lamb, J.; Jiménez, A.S.; Shafrir, A.; Sobey, M.; Van Tongeren, M.; et al. Prioritising action on occupational carcinogens in Europe: A socioeconomic and health impact assessment. Br. J. Cancer 2017, 117, 274-281. [CrossRef] [PubMed]

20. Tsai, R.; Luckhaupt, S.E.; Schumacher, P.; Cress, R.D.; Deapen, D.M.; Calvert, G.M. Acute myeloid leukemia risk by industry and occupation. Leuk. Lymphoma 2014, 55, 2584-2591. [CrossRef] [PubMed]

21. Spyratos, D.; Zarogoulidis, P.; Porpodis, K.; Tsakiridis, K.; Machairiotis, N.; Katsikogiannis, N.; Kougioumtzi, I.; Dryllis, G.; Kallianos, A.; Rapti, A.; et al. Occupational exposure and lung cancer. J. Thorac. Dis. 2013, 5 (Suppl. 4), S440-S445.

22. Ghittoni, R.; Accardi, R.; Chiocca, S.; Tommasino, M. Role of human papillomaviruses in carcinogenesis. Ecancermedicalscience 2015, 9. [CrossRef]

23. Shah, K.M.; Young, L.S. Epstein-Barr virus and carcinogenesis: Beyond Burkitt's lymphoma. Clin. Microbiol. Infect. 2009, 15, 982-988. [CrossRef]

24. Fung, J.; Lai, C.; Yuen, M. Hepatitis B and C virus-related carcinogenesis. Clin. Microbiol. Infect. 2009, 15, 964-970. [CrossRef]

25. Ministry of Health launches 'The National Periodic Health Screening' and 'Cancer Screening' Initiatives. Available online: https://www.mohap.gov.ae/en/MediaCenter/News/Pages/1381.aspx (accessed on 1 October 2019).

26. UAE Occupational Health and Safety Management System (Ohsms) National Standard; The National Emergency, Crisis and Disaster Management Authority: Abu Dhabi, United Arab Emirates, 2016.

27. Guidelines for Health and Safety in Workplace in the Federal Government; Federal Authority for Government Human Resources: Abu Dhabi, United Arab Emirates, 2020; updated.

28. Elobaid, Y.; Aw, T.-C.; Lim, J.N.; Hamid, S.; Grivna, M. Breast cancer presentation delays among Arab and national women in the UAE: A qualitative study. SSM-Popul. Health 2016, 2, 155-163. [CrossRef] [PubMed]

29. Daher, M. Cultural beliefs and values in cancer patients. Ann. Oncol. 2012, 23 (Suppl. 3), 66-69. [CrossRef] [PubMed]

30. Niederdeppe, J.; Levy, A.G. Fatalistic Beliefs about Cancer Prevention and Three Prevention Behaviors. Cancer Epidemiology Biomarkers Prev. 2007, 16, 998-1003. [CrossRef] [PubMed] 
31. Ryan, A.; Cushen, S.; Schellekens, H.; Ni Bhuachalla, E.; Burns, L.; Kenny, U.; Power, D.G. Poor Awareness of Risk Factors for Cancer in Irish Adults: Results of a Large Survey and Review of the Literature. Oncol. 2015, 20, 372-378. [CrossRef]

32. Elobaid, Y.; Aw, T.C.; Grivna, M.; Nagelkerke, N. Breast Cancer Screening Awareness, Knowledge, and Practice among Arab Women in the United Arab Emirates: A Cross-Sectional Survey. PLoS ONE 2014, 9, e105783. [CrossRef]

33. Pink Carvan. Available online: https://www.pinkcaravan.ae/ (accessed on 1 October 2019).

34. Incidence, Mortality and Prevalence by Cancer Site in United Arab Emirates; Global Cancer Observatory. WHO: Geneva, Switzerland, 2008.

35. Ministry of Health and Prevention Launches Cancer Awareness Program for Prevention and Early Detection of Colon Cancer; Ministro of Health and prevention: Abu Dhabi, United Arab Emirates, 2018.

36. Ramakant, P.; Singh, K.R.; Jaiswal, S.; Singh, S.; Ranjan, P.; Rana, C.; Jain, V.; Mishra, A.K. A Survey on Breast Cancer Awareness Among Medical, Paramedical, and General Population in North India Using Self-Designed Questionnaire: A Prospective Study. Indian J. Surg. Oncol. 2017, 9, 323-327. [CrossRef]

37. Gürdal, S.Ö.; Saraçoğlu, G.V.; Oran, E.Ş.; Yankol, Y.; Soybir, G.R. The effects of educational level on breast cancer awareness: A cross-sectional study in Turkey. Asian Pac. J. Cancer Prev. 2012, 13, 295-300. [CrossRef]

38. Marlow, L.A.; A Robb, K.; Simon, A.; Waller, J.; Wardle, J. Awareness of cancer risk factors among ethnic minority groups in England. Public Health 2012, 126, 702-709. [CrossRef]

39. Breslow, R.A.; Sorkin, J.D.; Frey, C.M.; Kessler, L.G. Americans' Knowledge of Cancer Risk and Survival. Prev. Med. 1997, 26, 170-177. [CrossRef]

40. Drewry, J.; Garcés-Palacio, I.C.; Scarinci, I. Awareness and Knowledge about Human Papillomavirus among Latina Immigrants. Ethn. Dis. 2010, 20, 327-333.

41. Inoue, M.; Iwasaki, M.; Otani, T.; Sasazuki, S.; Tsugane, S. Public awareness of risk factors for cancer among the Japanese general population: A population-based survey. BMC Public Health 2006, 6, 2. [CrossRef] [PubMed]

42. Cervical Cancer Deaths in UAE Doubled in 6 Years: Study. 2019. Available online: https://www.khaleejtimes. com/news/uae-health/cervical-cancer-deaths-in-uae-doubled-in-6-years-study (accessed on 7 October 2019).

43. Bates, S.; Holmes, J.; Gavens, L.; De Matos, E.G.; Li, J.; Ward, B.; Hooper, L.; Dixon, S.; Buykx, P. Awareness of alcohol as a risk factor for cancer is associated with public support for alcohol policies. BMC Public Health 2018, 18, 688. [CrossRef] [PubMed]

44. Matthee, R. Alcohol in the Islamic Middle East: Ambivalence and Ambiguity. Past Present. 2014, 222, $100-125$. [CrossRef]

45. Tobacco and Alcohol Provisions; Department of Economic Development: Dubai, United Arab Emirates, 2009.

46. Anti-Smoking Initiatives by Hospitals and Health Ministries in UAE; Etisalat UAE: Abu Dhabi, United Arab Emirates, 2019.

47. UAE Health Ministry Continues Efforts to Curb Smoking; Dubai Media Incorporated: Dubai, United Arab Emirates, 2019.

48. Milcarz, M.; Polańska, K.; Bąk-Romaniszyn, L.; Kaleta, D. Tobacco Health Risk Awareness among Socially Disadvantaged People-A Crucial Tool for Smoking Cessation. Int. J. Environ. Res. Public Health 2018, 15, 2244. [CrossRef] [PubMed]

49. Vallès, Y.; Inman, C.K.; Peters, B.; Wareth, L.A.; Abdulle, A.; Alsafar, H.; Al Anouti, F.; Al Dhaheri, A.; Galani, D.; Haji, M.; et al. Incense Burning is Associated with Human Oral Microbiota Composition. Sci. Rep. 2019, 9, 10039. [CrossRef] [PubMed]

50. Amd, F.A.M.A.; Ahmed, H.G. Assessment of awareness towards some carcinogens in hail region, saudia carcinogens in hail region, saudia carcinogens in hail region, saudia arabi. Manag. Health 2013, XVII, 15-18.

51. Mansour, D.; Nashwan, A.; Abu Rasheed, H.; Hararah, M.; Nassar, H.; Abu Abbas, R.; Alnuaimi, M.; Mrayat, B. Use of Social Media in Breast Cancer Awareness: GCC Countries' Experience. J. Glob. Oncol. 2018, 4 (Suppl. 2), 30s. [CrossRef]

52. Gulf Cancer Awareness Week. Available online: https://www.mohap.gov.ae/en/MediaCenter/News/Pages/ 1501.aspx (accessed on 1 October 2019).

(C) 2020 by the authors. Licensee MDPI, Basel, Switzerland. This article is an open access article distributed under the terms and conditions of the Creative Commons Attribution (CC BY) license (http://creativecommons.org/licenses/by/4.0/). 